\title{
A Síndrome de Burnout em professores médicos durante a pandemia da Covid-19
}

\author{
Burnout Syndrome in medical teachers during the Covid-19 pandemic
}

Síndrome de Burnout en profesores de medicina durante la pandemia Covid-19

\begin{abstract}
Edson Satoshi Miyazato ${ }^{1 *}$, João Pedro Nakamura Amaral ${ }^{1}$, Albert Schiaveto de Souza ${ }^{1}$, Anderson Ravy Stolf ${ }^{1}$.
\end{abstract}

\section{RESUMO}

Objetivo: Investigar a prevalência e os fatores associados ao Burnout em professores médicos durante a pandemia da Covid-19 e correlacioná-los com suas áreas da vida no trabalho. Métodos: Trata-se de um estudo transversal, realizado em setembro de 2020, com 39 professores médicos de uma Escola de Medicina que preencheram um questionário online contendo o Inventário de Burnout de Maslach, Áreas da Vida no Trabalho, além de dados sociodemográficos. Os dados obtidos foram analisados com auxílio do sof tware IBM SPSS Statistics. O estudo foi aprovado por Comitê de Ética em Pesquisa (protocolo 4.255.791). Resultados: A prevalência encontrada foi de $40 \%$ de Burnout em professores médicos, dos quais os homens apresentaram maior realização pessoal e as mulheres tendenciaram à maior exaustão emocional. A sensação de sobrecarga no trabalho não influenciou na exaustão emocional, tampouco na realização pessoal. Quanto mais locais de trabalho, maiores foram os escores de exaustão emocional e despersonalização dos professores. Conclusão: A pandemia da Covid-19 trouxe mudanças na forma de trabalho, necessitando de adaptações. Sugere-se que possuir menos locais de trabalho pode ser uma prática recomendável ao docente médico e há benefício em avaliar e gerenciar os processos de esgotamento no corpo docente médico.

Palavras-chave: Covid-19, Esgotamento profissional, Educação médica, Faculdades de medicina, Docentes de medicina.

\section{ABSTRACT}

Objective: To investigate the prevalence and associated factors of burnout in medical professors during the Covid-19 pandemic and correlate them with their Areas of worklife. Methods: This is a cross-sectional study, carried out in September 2020, with 39 medical professors from a School of Medicine who completed an online questionnaire containing the Maslach Burnout Inventory, Areas Worklife Questionnaire and sociodemographic data. The data obtained were analyzed by the IBM SPSS Statistics software. The study was approved by the Research Ethics Committee ((protocol 4.255.791). Results: The prevalence of Burnout was $40 \%$ in medical professors. There was greater personal accomplishment in men and women tended to have greater emotional exhaustion. Workload did not influence emotional exhaustion or personal accomplishment. More workplaces are related to higher emotional exhaustion and depersonalization scores. Conclusion: The Covid-19 pandemic has brought changes in the work way, requiring adaptations. It is suggested that having fewer workplaces is a recommended practice for medical faculty and there is benefit in evaluating and managing the exhaustion processes in the medical faculty.

Key words: Covid-19, Professional burnout, Medical education, University professor, Medical school.

1 Universidade Federal do Mato Grosso do Sul (UFMS). Campo Grande - MS.

*E-mail: edson_satoshi@hotmail.com

SUBMETIDO EM: 12/2021

ACEITO EM: 1/2022

PUBLICADO EM: 1/2022 


\section{RESUMEN}

Objetivo: Investigar la prevalencia y factores asociados al Burnout en profesores de medicina durante la pandemia de Covid-19 y correlacionarlos con sus áreas de la vida laboral. Métodos: Se trata de un estudio transversal, realizado en septiembre de 2020, con 39 profesores de medicina de una Facultad de Medicina que completaron un cuestionario en línea que contenía el Inventario de Burnout Maslach, Áreas de la Vida en el Trabajo, además de datos sociodemográficos. Los datos obtenidos se analizaron mediante el software IBM SPSS Statistics. El estudio fue aprobado por el Comité de Ética en Investigación (protocolo 4.255.791). Resultados: La prevalencia encontrada fue del $40 \%$ de Burnout en los profesores de medicina, de los cuales los hombres mostraron mayor realización personal y las mujeres tendieron a un mayor agotamiento emocional. La sensación de sobrecarga laboral no influyó en el agotamiento emocional ni en la realización personal. Cuantos más lugares de trabajo, mayor es la puntuación de agotamiento emocional y despersonalización de los profesores. Conclusión: La pandemia de Covid-19 trajo cambios en la forma de trabajar, requiriendo adaptaciones. Se sugiere que tener menos lugares de trabajo es una práctica recomendada para los profesores de medicina y hay un beneficio en evaluar y manejar los procesos de agotamiento en la facultad de medicina.

Palabras clave: Covid-19, Burnout profesional, Educación médica, Facultades de medicina, Profesores de medicina.

\section{INTRODUÇÃO}

O novo coronavírus em 2019 (Covid-19) causou um grande impacto sobre os sistemas hospitalares e as equipes de saúde, com pressão física e emocional dos profissionais na linha de frente. Dentre os médicos, já considerada uma profissão exigente e estressante, a rotina exaustiva, a exposição contínua ao estresse crônico onde precisam lidar com intensas responsabilidades com os pacientes e seus familiares têm gerado impactos em sua saúde mental, contribuindo para o agravamento do estresse físico e emocional, ansiedade e Burnout (AYDIN SAYILAN A, et al., 2021; RAUDENSKÁ J, et al., 2020).

O Burnout é descrito como uma reação à tensão emocional crônica no trabalho, que pode ocorrer quando se lida excessivamente com pessoas (TIJDINK JK, et al., 2014). Assim, reconhece-se cada vez mais como um fator que não afeta apenas a saúde do médico, mas também dos pacientes sob seus cuidados (AMANULLAH S e SHANKAR RR, 2020).

Antes mesmo da pandemia da Covid-19, nota-se que o Burnout é um problema comum entre os médicos (RESTAURI N e SHERIDAN AD, 2020; AMANULLAH S e SHANKAR RR, 2020). Dentre os que desempenham funções clínicas e também são professores (considerados de certa maneira o centro da comunidade médica, pois atuam como educadores, gestores e, talvez o mais importante, como modelos para estudantes, residentes e colegas) notam-se o interesse na educação, mas muitos se sentem incapazes de despender energia suficiente ao ensino, por estarem sob pressão, falta de tempo e, ainda, tornando -os vulneráveis ao estresse e esgotamento relacionados ao seu trabalho (TIJDINK JK, et al., 2014; REED DA, et al., 2007).

O Burnout nas faculdades médicas, além de prejudicar a educação de seus acadêmicos, também pode causar o comprometimento no atendimento ao paciente, aumento de erros médicos e condições adversas (RAO SK, et al., 2017; YU J, et al., 2019). Assim, o trabalho do professor médico pode gerar repercussões à saúde mental, influenciando negativamente em sua qualidade de vida e também na qualidade de ensino de seus alunos (ROTENSTEIN LS, et al., 2018; NASSAR AK, et al., 2020; DE ARAÚJO LEITE TIA, et al., 2019; YU J, et al., 2019).

Os levantamentos demonstram a carência de dados científicos relacionados ao docente médico e, também, em tempos da Covid-19, tema do presente estudo. O objetivo do estudo foi levantar a prevalência de docentes médicos com sintomas de Burnout na vigência da pandemia da Covid-19 e correlacioná-los com suas áreas da vida no trabalho, entre suas adequações profissionais e seu ambiente de trabalho. 


\section{MÉTODOS}

Realizou-se um estudo transversal com o corpo docente médico de uma Faculdade de Medicina do Mato Grosso do Sul / Brasil, sendo ele exclusivo ou não da instituição, de qualquer idade, especialidade ou carga horária de trabalho na instituição, na população de 80 docentes exclusivamente médicos, onde foram convidados e enviados questionários de forma online, em setembro/2020, para participação do estudo. $O$ questionário não incluiu o nome para preenchimento, o consentimento foi obtido por todos os participantes e os dados mantidos em sigilo.

Em relação aos instrumentos de pesquisa, utilizou-se um questionário de dados sociodemográficos e laborais, que incluiu dados de gênero, idade, estado civil, tempo de trabalho na instituição, comorbidades psiquiátricas e regime de trabalho na instituição. Para a avaliação do Burnout, utilizou-se o Inventário de Burnout de Maslach [Maslach Burnout Inventory - Human Services Survey for Medical Personnel, MBIHSS(MP)], instrumento mais usado para se avaliar como o trabalhador vivencia seu trabalho e validado no país (PEREIRA SS, et al., 2021).

A escala é composta por 22 itens, cada pergunta apresenta resposta em escala de frequência de até sete pontos que vai de zero (nunca) até seis (diariamente). São nove itens de Exaustão Emocional (EE), cinco itens de Despersonalização (DP) ou Distanciamento Afetivo, e oito itens de Realização Profissional (PA). Avalia-se os índices de cada dimensão, sendo as maiores pontuações para exaustão e despersonalização e baixas pontuações para realização profissional indicadores para o Burnout. Os limites estabelecid os para as dimensões são alta EE $>=27$, alta DP $>=10$ e baixa PA $<=33$ (MASLACH C, et al., 2018). Outra forma de cálculo também usado é o Burnout clinicamente significativo ("burnout overall"), definido como alto $E E>=27$ e/ou alta DP >= 10 (DUKE NN, et al., 2020; WINDOVER AK, et al., 2018). Calculou-se ambas as definições, para comparação com outros estudos.

Sobre as áreas da vida no trabalho, utilizou-se o questionário Areas of worklife Survey (AWS), de Leiter MP e Maslach C (2004), estudado e validado também no país por Porto A (2019), onde visa avaliar seis dimensões no contexto e conteúdo no trabalho, que propõe quanto maior a lacuna percebida entre a pessoa e o trabalho, maior a probabilidade de Burnout, assim como o inverso, de quanto maior a consistência, maior seria o seu engajamento.

A avaliação da correlação linear entre os escores de Burnout em cada dimensão da escala MBI-HSS (MP) e os escores nas questões da escala AWS foi realizada por meio do teste de correlação linear de Pearson. Já a comparação entre sexos ou entre docentes de diferentes faixas etárias, nos escores das diferentes dimensões da escala MBI-HSS (MP), foi realizada por meio do teste t-student (sexos) ou Análise de Variância (ANOVA) de uma via (faixas etárias). Os demais resultados deste estudo foram apresentados na forma de estatística descritiva. A análise estatística foi realizada no programa IBM SPSS Statistics., versão 23.0, considerando um nível de significância de $5 \%$.

O estudo foi autorizado pelo Comitê de Ética em Pesquisa (CEP) da Universidade Federal do Mato Grosso do Sul, sob protocolo 4.255.791 e CAAE 29986819.1.0000.0021.

\section{RESULTADOS}

Um total de $39(48,75 \%)$ dos 80 docentes médicos responderam completamente ao questionário. A maioria (20; $51,3 \%)$ foram homens, entre 45 e 54 anos (15; 38,5\%), com renda mensal entre 11 a 20 salários mínimos (14; $35,9 \%)$, de maioria étnica branca $(36 ; 92,31 \%)$, casados $(31 ; 79,49 \%)$ e com regime parcial de 20 horas semanais na instituição (26; 66,67\%). Os dados demográficos estão apresentados na Tabela 1. 
Tabela 1 - Características demográficas dos participantes, n=39. 2020.

\begin{tabular}{|c|c|c|}
\hline Variável & $\%$ & $\mathbf{N}$ \\
\hline \multicolumn{3}{|l|}{ Gênero } \\
\hline Masculino & $51.3 \%$ & 20 \\
\hline Feminino & $48.7 \%$ & 19 \\
\hline \multicolumn{3}{|l|}{ Etnia } \\
\hline Asiático(a) ou procedente das ilhas do Pacífico & $2.56 \%$ & 1 \\
\hline $\operatorname{Branco}(\mathrm{a})$ & $92.31 \%$ & 36 \\
\hline Mais de uma etnia & $5.13 \%$ & 2 \\
\hline \multicolumn{3}{|l|}{ Faixa etária } \\
\hline Entre 25 e 34 anos & $2.6 \%$ & 1 \\
\hline Entre 35 e 44 anos & $30.8 \%$ & 12 \\
\hline Entre 45 e 54 anos & $38.5 \%$ & 15 \\
\hline Entre 55 e 64 anos & $20.5 \%$ & 8 \\
\hline Entre 65 e 74 anos & $7.7 \%$ & 3 \\
\hline \multicolumn{3}{|l|}{ Estado civil } \\
\hline Casado(a) & $79.49 \%$ & 31 \\
\hline Viúvo(a) & $2.56 \%$ & 1 \\
\hline Divorciado(a) & $12.82 \%$ & 5 \\
\hline Em uma união estável ou casamento civil & $5.13 \%$ & 2 \\
\hline \multicolumn{3}{|l|}{ № de locais de trabalho } \\
\hline 1 & $7.69 \%$ & 3 \\
\hline 2 & $30.77 \%$ & 12 \\
\hline 3 & $46.15 \%$ & 18 \\
\hline 4 & $10.26 \%$ & 4 \\
\hline 5 ou mais & $5.13 \%$ & 2 \\
\hline \multicolumn{3}{|l|}{ Regime de trabalho } \\
\hline Parcial (20 horas/semana) & $66.67 \%$ & 26 \\
\hline Integral (40 horas/semana) & $33.33 \%$ & 13 \\
\hline \multicolumn{3}{|l|}{ Renda mensal } \\
\hline Até 10 salários mínimos & $2.6 \%$ & 1 \\
\hline De 11 a 20 salários mínimos & $35.9 \%$ & 14 \\
\hline De 21 a 30 salários mínimos & $30.8 \%$ & 12 \\
\hline De 31 a 40 salários mínimos & $25.6 \%$ & 10 \\
\hline De 41 a 50 salários mínimos & $5.1 \%$ & 2 \\
\hline \multicolumn{3}{|l|}{ Acompanhamento com psicólogo } \\
\hline Sim & $12.18 \%$ & 5 \\
\hline Não & $87.82 \%$ & 34 \\
\hline \multicolumn{3}{|l|}{ Acompanhamento com psiquiatra } \\
\hline Sim & $10,26 \%$ & 4 \\
\hline Não & $89.74 \%$ & 35 \\
\hline \multicolumn{3}{|l|}{ Uso contínuo de medicamento psiquiátrico } \\
\hline Sim & $20.51 \%$ & 8 \\
\hline Não & $79.49 \%$ & 31 \\
\hline \multicolumn{3}{|l|}{ Já apresentou doença psiquiátrica } \\
\hline $\operatorname{Sim}$ & $23.08 \%$ & 9 \\
\hline Não & $76.92 \%$ & 30 \\
\hline \multicolumn{3}{|l|}{ Apresenta atualmente doença psiquiátrica } \\
\hline Sim & $7.69 \%$ & 3 \\
\hline Não & $92.31 \%$ & 36 \\
\hline
\end{tabular}

Fonte: Miyazato ES, et al., 2022. 
Para a avaliação do Burnout, utilizou-se a escala de MBI-HSS (MP) e seus domínios, apresentados na Tabela 2. No cálculo para critérios de Burnout clinicamente significativo, encontrou-se uma prevalência de $40 \%$ entre os docentes.

Tabela 2 - Análise descritiva dos domínios de Burnout, MBI-HSS (MP). 2020.

\begin{tabular}{lccccccc}
\hline Dimensão & Média $\pm \mathbf{D P}$ & \multicolumn{2}{c}{ Baixo N(\%) } & \multicolumn{2}{c}{ Moderado N(\%) } & \multicolumn{3}{c}{ Alto N(\%) } \\
\hline Exaustão Emocional $^{*}$ & $19.13 \pm 13,73$ & 21 & $53,8 \%$ & 7 & $17,9 \%$ & 11 & $28,2 \%$ \\
Despersonalização $^{* *}$ & $4.87 \pm 5.03$ & 27 & $69,2 \%$ & 8 & $20,5 \%$ & 4 & $10,3 \%$ \\
Realização Pessoal $^{* * *}$ & $41.69 \pm 5.62$ & 1 & $2,6 \%$ & 10 & $25,6 \%$ & 28 & $71,8 \%$ \\
\hline
\end{tabular}

Legenda: MBI - Human Services Survey - MBI-HSS: Copyright (@1981 Christina Maslach \& Susan E. Jackson. All rights reserved in all media. Published by Mind Garden, Inc., www.mindgarden.com; *Escores de Exaustão emocional: <17, 17 a 26, $>26$ são categorizados como baixo, moderado e alto respectivamente; ${ }^{* *}$ Escores de Despersonalização: $<7,7$ a $12,>12$ são categorizados como baixo, moderado e alto respectivamente; ${ }^{* * *}$ Escores de Realização pessoal: $<32,32$ a 38, >38 são categorizados como baixo, moderado e alto respectivamente; MBI-HSS (MP): Maslach Burnout Inventory-Human Services Survey (Medical Personnel); DP: Desvio padrão.

Fonte: Miyazato ES, et al., 2022.

Para a avaliação das Áreas da vida no trabalho, foi utilizada a escala AWS, composta por 28 itens, onde cada pergunta possui resposta em escala de frequência tipo Likert de 5 pontos, que vai de 1 (discordo fortemente) até 5 (concordo fortemente). A escala contém 5 itens de carga de trabalho, 4 itens de controle, 5 itens de recompensa, 5 itens de comunidade, 6 itens de justiça e 4 itens de valores. Para cada uma das subescalas, quanto maior a pontuação, maior o grau de congruência entre o local de trabalho e as preferências do entrevistado, enquanto uma menor pontuação demonstra uma incongruência entre o trabalhador e seu local de trabalho. A "Justiça" foi a única área no estudo que demonstrou incongruência (Tabela 3).

Tabela 3 - Escores do AWS, Áreas da vida no trabalho. 2020.

\begin{tabular}{|c|c|}
\hline Questão & Média土EPM \\
\hline \multicolumn{2}{|l|}{ Carga de Trabalho } \\
\hline Ex.: Eu não tenho tempo para fazer o trabalho que precisa ser feito & $3,14 \pm 0,15$ \\
\hline \multicolumn{2}{|l|}{ Controle } \\
\hline Ex.: Eu tenho controle sobre como eu faço meu trabalho & $3,21 \pm 0,10$ \\
\hline \multicolumn{2}{|l|}{ Recompensa } \\
\hline Ex.: Eu recebo reconhecimento dos outros pelo meu trabalho & $3,41 \pm 0,12$ \\
\hline \multicolumn{2}{|l|}{ Comunidade } \\
\hline $\begin{array}{l}\text { Ex.: Os membros do meu grupo de trabalho se comunicam abertamente } \\
\text { Justiça }\end{array}$ & $3,26 \pm 0,12$ \\
\hline Ex.: Os recursos são alocados de forma justa aqui & $2,53 \pm 0,10$ \\
\hline \multicolumn{2}{|l|}{ Valores } \\
\hline Ex.: Os meus valores e os valores da organização são semelhantes & $3,30 \pm 0,08$ \\
\hline
\end{tabular}
Legenda: AWS Instrument - Copyright (C 2000, 2011 by Michael P. Leiter \& Christina Maslach. All rights reserved in all media. Published by Mind Garden, Inc., www.mindgarden.com; EPM: Erro padrão médio. Fonte: Miyazato ES, et al., 2022.

O estudo também comparou as dimensões do Burnout com os domínios das Áreas da Vida no Trabalho (AWS) e a quantidade de locais de trabalho que os docentes atuam (Tabela 4). Encontrou-se correlações negativas entre carga de trabalho $x$ exaustão emocional (correlação moderada, $r=-0.600 ; p<0.001$ ), controle $x$ exaustão emocional (fraca, $r=-0.322 ; p=0.046$ ), recompensa $x$ despersonalização (fraca, $r=-0.320 ; p=0.047$ ) e correlações positivas entre controle $x$ realização pessoal (fraca, $r=0.349 ; p=0.029$ ), carga de trabalho $x$ realização pessoal (moderada, $r=0.529 ; p=0.001$ ), quantidade de locais de trabalho $x$ exaustão emocional (moderada, $r=0.534 ; p<0.001$ ) e quantidade de locais de trabalho $x$ despersonalização (moderada, $r=0.511$; $\mathrm{p}=0.001$ ). 
Tabela 4 - Correlações do Burnout com AWS e Quantidade de Locais de trabalho. 2020.

\begin{tabular}{|c|c|c|c|}
\hline Correlações & $\begin{array}{c}\text { Exaustão } \\
\text { Emocional }\end{array}$ & Despersonalização & Realização Pessoal \\
\hline Carga de trabalho & $\begin{array}{l}r=-0.600 \\
(p<0.001)\end{array}$ & $\begin{array}{l}r=-0.251 \\
(p=0.123)\end{array}$ & $\begin{array}{c}r=0.529 \\
(p=0.001)\end{array}$ \\
\hline Controle & $\begin{array}{c}r=-0.322 \\
(p=0.046)\end{array}$ & $\begin{array}{c}r=-0.313 \\
(p=0.052)\end{array}$ & $\begin{array}{c}r=0.349 \\
(p=0.029)\end{array}$ \\
\hline Recompensa & $\begin{array}{l}r=-0.190 \\
(p=0.247)\end{array}$ & $\begin{array}{l}r=-0.320 \\
(p=0.047)\end{array}$ & $\begin{array}{c}r=0.142 \\
(p=0.388)\end{array}$ \\
\hline Comunidade & $\begin{array}{c}r=-0.200 \\
(p=0.222)\end{array}$ & $\begin{array}{c}r=-0.187 \\
(p=0.255)\end{array}$ & $\begin{array}{c}r=0.208 \\
(p=0.205)\end{array}$ \\
\hline Justiça & $\begin{aligned} & r=0.000 \\
&(p=0.999)\end{aligned}$ & $\begin{array}{c}r=0.039 \\
(p=0.812)\end{array}$ & $\begin{array}{l}r=-0.038 \\
(p=0.819)\end{array}$ \\
\hline Valores & $\begin{array}{c}r=-0.063 \\
(p=0.704)\end{array}$ & $\begin{array}{c}r=-0.152 \\
(p=0.355)\end{array}$ & $\begin{array}{c}r=0.145 \\
(p=0.379)\end{array}$ \\
\hline Quantidade de locais de trabalho & $\begin{array}{c}r=0.534 \\
(p<0.001)\end{array}$ & $\begin{array}{c}r=0.511 \\
(p=0.001)\end{array}$ & $\begin{array}{l}r=-0.275 \\
(p=0.091)\end{array}$ \\
\hline
\end{tabular}

Legenda: $r=$ coeficiente de correlação linear de Pearson.

Fonte: Miyazato ES, et al., 2022.

Na comparação entre gêneros, houve maior sensação de realização pessoal entre os homens $(p=0.044)$ e tendência de maior exaustão emocional entre as mulheres $(p=0.056)$. Em relação a idade, não se encontrou diferenças quando comparadas as faixas etárias (Tabela 5).

Tabela 5 - Comparações entre os domínios de Burnout com gênero e faixa etária. 2020.

\begin{tabular}{|c|c|c|c|c|c|c|c|}
\hline Dimensão & $\begin{array}{c}\text { Masculino } \\
\text { Média } \pm \text { EPM }\end{array}$ & $\begin{array}{c}\text { Feminino } \\
\text { Média } \pm E P M\end{array}$ & p & $\begin{array}{c}\leq 44 \text { anos } \\
\text { Média } \pm \text { EPM }\end{array}$ & $\begin{array}{l}\text { 45-54 anos } \\
\text { Média } \pm \text { EPM }\end{array}$ & $\begin{array}{c}\geq 55 \text { anos } \\
\text { Média } \pm \text { EPM }\end{array}$ & $\mathbf{p}$ \\
\hline & 15 & 2 & ${ }^{*}$ & 22 & 2 & $1=$ & $5^{\dagger}$ \\
\hline Desperson & 4 & 39 & $2<$ & $6.54 \pm 1.67$ & 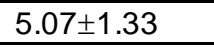 & 2.64 & $65^{\dagger}$ \\
\hline $\begin{array}{l}\text { Realização } \\
\text { Pessoal }\end{array}$ & $43.45 \pm 1.35$ & $39.84 \pm 1.38$ & $0.044^{*}$ & $40.54 \pm 1.60$ & $40.93 \pm 1.60$ & $41.69 \pm 0.90$ & $0.250^{\dagger}$ \\
\hline
\end{tabular}

Legenda: *Teste T para igualdade de médias; †ANOVA.

Fonte: Miyazato ES, et al., 2022.

\section{DISCUSSÃO}

Os domínios do MBI no presente estudo revelaram que $28,2 \%$ dos docentes médicos apresentaram altos níveis de exaustão emocional, 10,3\% tiveram altos níveis de despersonalização e 71,8\% apresentaram alta realização pessoal. A maioria dos estudos que avaliam os níveis de exaustão emocional em docentes médicos, em período fora da pandemia, apresentam resultados que variam entre 25 e $36 \%$ (WINDOVER AK, et al., 2018; ARVANDI Z, et al., 2016; VUKMIROVIC M, et al., 2020; CHICHRA A, et al., 2019; DUKE NN, et al., 2020; GANESHAN D, et al., 2019; YU J, et al., 2019). Durante a pandemia, um estudo de Giusti EM, et al. (2020) encontrou 31,9\% de altos níveis de exaustão emocional, 12,1\% de altos níveis de despersonalização e $34,3 \%$ de alta realização pessoal em profissionais da saúde em geral.

Quanto ao Burnout clinicamente significativo, encontrou-se uma prevalência de $40 \%$ entre os docentes, valores próximos a outros estudos em períodos pré-pandemia, com 37,91\% de Jinli Y, et al. (2019) e 35\% de Windover AK, et al. (2018). Durante a pandemia, a prevalência encontrada nos estudos que utilizaram o MBI foi de $76 \%$ entre os residentes médicos, no estudo de Dimitriu MCT, et al. (2020) e 39\% em profissionais de saúde em geral (WU Y, et al., 2020).

Em relação a despersonalização, 10,3\% dos docentes médicos apresentaram altos níveis, ficando abaixo das demais médias de outros estudos que variaram de 5,6\% a 24\% (GIUSTI EM, et al., 2020; CHICHRA A, et al., 2019; DUKE NN, et al., 2020; GANESHAN D, et al., 2019; JINLI, et al., 2019). Quanto a realização 
pessoal, os estudos em períodos pré-pandemia variaram entre $10,21 \%$ de Jinli $Y$, et al.(2019) a $52 \%$ de Ganeshan D, et al. (2019) na população médica docente entrevistada, e outro estudo de Giusti EM, et al. (2020) durante a Covid-19 apresentou 34,3\%comparado com $71,8 \%$ de alta realização no presente estudo. Embora os docentes do estudo sejam muito realizados, é importante salientar que não se obteve 100\% de participação dos mesmos, e as amostras foram obtidas no contexto de valorização do profissional da saúde frente à pandemia da Covid-19.

Um interessante achado foi a relação entre quanto maior sensação de carga de trabalho, menor o nível de exaustão emocional (correlação moderada, Pearson 0.600. $p=0.001$ ). Uma das explicações para a relação é que a sensação da carga de trabalho pode ser influenciada pela realização pes soal, já que nenhum docente apresentou baixa realização, mesmo tendo percebido, também, sua alta carga (correlação moderada, Pearson 0.529. $p=0.001$ ). Sabe-se que o estresse pode af etar negativamente a saúde mental e a satisfação no seu emprego, mas parece não af etar de forma considerável sua satisfação em geral (SCHINDLER BA, et al., 2006). Assim, ter uma alta percepção de "estar satisfeito no trabalho" pode ser um fator protetor (CHICHRA A, et al., 2019; ARVANDI Z, et al., 2016; DUKE NN, et al., 2020).

Outros fatores como ter segurança do emprego, sensações de que sua experiência profissional está sendo usada ao máximo, a própria figura do médico e sua posição também são fontes de relatos de alta satisf ação (CHICHRA A, et al., 2019). Em estudos auto-referidos fora da pandemia encontramos dados de médicos se sentirem sob estresse e não relatarem sintomas de Burnout, como no estudo de Dandar VM, et al. (2019), embora também haja correlação entre Burnout e exaustão emocional, visto no estudo de Knox M, et al. (2018), porém sem o uso de instrumentos validados.

Quando comparados os gêneros, houve tendência de mulheres terem maior exaustão emocional que os homens, sem diferença estatística (Tabela 5), acompanhando a tendência em outros estudos, como os de Chichra A, et al. (2019) e Duke NN, et al. (2020), embora outros apontem uma maior exaustão entre as mulheres, apontados em Dandar VM, et al. (2019) e em Nassar AK, et al. (2020), com mais responsabilidades domésticas do que os homens, o que pode ter contribuído para os tais achados e também influenciar nos níveis de realização pessoal entre os gêneros, onde mulheres se sentiram menos realizadas que os homens $(p=0.044)$. Durante a pandemia da Covid-19, Kanampallill TG, et al. (2020) apontou maiores níveis de Burnout em residentes médicas, com estressores específicos o cuidado com crianças e o balanço entre vida pessoal e profissional. Uma revisão sistemática, inclusive, prevê que o impacto do Burnout nas mulheres durante a pandemia deve ser considerável e duradouro (AMANULLAH S e SHANKAR RR, 2020).

Em relação à faixa etária, não houve diferença significativa entre os níveis de Burnout e do AWS, como nos estudos pré-pandemia de Arvandi Z, et al. (2016) e Vukmirovic M, et al. (2020), mas pode ser encontrado maiores níveis em jovens, que relataram alta percepção de estresse no trabalho, devido a interações com pacientes e residentes. A relação entre Burnout e anos de trabalho também não foi encontrada. A menor experiência, a sobrecarga cognitiva, a ansiedade pelo bom desempenho e o aprendizado para lidar com as demandas poderiam ser alguns dos fatores que explicariam a tendência de alguns estudos referirem maior sobrecarga de trabalho entre os mais jovens, apontados por Nassar AK, et al. (2020), Chichra A, et al. (2019), Leite TIA, et al. (2019), mas que não foi detectado pelo presente estudo.

Este estudo também revelou que, quanto mais locais de trabalho, maiores são a exaustão emocional (moderado, Pearson 0.534. $p<0.001$ ) e a despersonalização (moderado, Pearson 0,511. $p=0,001$ ), o que era esperado, pois os professores em geral, não somente médicos, tem dupla jornada, com longas horas de trabalho, sobrecarga e assim, sofrem de exaustão (LEITE TIA, et al., 2019). Para o ensino, pode-se afirmar que possuir menos locais de trabalho é uma prática recomendável, a fim de diminuir o esgotamento e o distanciamento af etivo dos docentes médicos com os alunos.

É importante salientar que a despersonalização e a exaustão emocional são fatores de risco para demissões e af eta diretamente no cuidado ao paciente, podendo inclusive aumentar reclamações em setores como o da Ouvidoria. Para o paciente insatisfeito, a despersonalização do médico parece influenciar mais do que própria exaustão emocional (WINDOVER AK, et al., 2018). 
Os docentes médicos que também prestam cuidados aos pacientes normalmente possuem maiores níveis de estresse, se comparado a outros que ficam somente em sala de aula ou em pesquisa (DANDAR VM, et al., 2019). Estudos também mostram que os serviços administrativos podem causar mais estresse que 0 próprio cuidado ao paciente, onde o tempo gasto em tarefas administrativas causam menor satisfação na carreira, maiores níveis de Burnout e tendência a atender menos pacientes (RAO SK, et al., 2017; NASSAR AK, et al., 2020).

Em relação às Áreas da vida no trabalho, o AWS foi criado para avaliar as dimensões do trabalho de um indivíduo, sendo suas dimensões a carga de trabalho, controle, recompensa, comunidade, justiça e valores. Os indivíduos que possuem bom engajamento em tais dimensões tendem a possuir fatores protetores contra o Burnout devido ao envolvimento e eficácia profissional em sua instituição (LEITER MP e MASLACH C, 2011). Assim, o AWS pode identificar aspectos problemáticos nas formas de trabalho e também fornecer dados para um melhor direcionamento às resoluções de conflitos e pontos incongruentes.

Dentre as dimensões avaliadas, a Justiça foi a única dimensão que apontou mais incongruência dos docentes médicos em nossa instituição: $59 \%$ discordam que os recursos são alocados de forma justa, $66,7 \%$ discordam que as oportunidades são decididas exclusivamente com base em mérito e 51,3\% discordam que haja procedimentos eficazes disponíveis quando se questiona se uma decisão foi justa. Assim, sentir-se injustiçado pode comprometer a relação do indivíduo com sua organização (LEITER MP e MASLACH C, 2004). Processos que demonstrem maior transparência, indicações baseadas em meritocracia nos programas de seleção interna para cargos e ampla divulgação de ações da instituição poderiam ser úteis para atenuar e esclarecer os sentimentos em nossos docentes. Os valores, por último, representam quanto os valores individuais estão de acordo com os valores de sua organização, aproximando (ou distanciando) o indivíduo dos valores centrais de sua organização, havendo congruência em nosso estudo.

No contexto da Covid-19, os novos procedimentos, regras e protocolos, assim como a insegurança de em qualquer momento serem infectados pelo vírus geram fontes de esgotamento (WU Y, et al., 2020). Isso poderia gerar um impacto negativo, piorando e reduzindo a capacidade dos sistem as de saúde de lidar com as demandas (GIUSTI EM, et al., 2020). Para o paciente, o aumento de erros médicos comprometeria a qualidade do serviço médico e a segurança do paciente. Sabe-se que o esgotamento também pode levar à negligência e aumentar os riscos de infecção, e a própria equipe sofrer consequências como o desenvolvimento de síndromes depressivas, pensamentos suicidas e até doenças físicas (DIMITRIU MCT, et al., 2020).

Assim, formas de prevenir e diminuir o Burnout devem ser aprimoradas. Programas de prevenção, of ertas de serviços psiquiátricos, psicológicos (inclusive anônimos) e de apoio, como creches, podem impactar a vida dos profissionais de saúde (KANNAMPALLIL TG, et al., 2020). Práticas como atenção plena (Minfulness), que aborda qualidades específicas da atenção e a consciência das experiências no momento presente, por meio da meditação, tem demonstrado bons resultados, onde sua prática em programas dentro de hospitais ajudou a reduzir o impacto da mudança organizacional nos médicos e os participantes relataram que lidam melhor com o Burnout, inclusive demonstrando redução ef etiva no esgotamento através da autoconsciência e a atenção plena (AMANULLAH S e SHANKAR RR, 2020).

Outras abordagens também podem ser úteis, como Terapia Cognitivo-Comportamental (TCC) focada no trauma, que já demonstrou ser útil em epidemias anteriores, e também o apoio social e dos cônjuges como sendo um importante fator de proteção sobre o trauma e a saúde mental (DIMITRIU MCT, et al., 2020). Outras recomendações a serem consideradas incluem a realização um programa oficial voltado à redução de redução do Burnout of erecido dentro da instituição, acessos online ou por telefone a linhas de apoio que of ereçam suporte em saúde mental, programas para os cônjuges e depend entes, educação médica voltada ao gerenciamento de estresse e esgotamento (AMANULLAH S e SHANKAR RR, 2020).

Em relação ao escopo acadêmico, o Burnout reduz diretamente a prontidão para mudar, por isso de sua importância nos processos de mudanças administrativas ou em grades curriculares (ARVANDI Z, et al., 2016). Estando menos sobrecarregado, o docente médico poderá estar mais aberto a novas ideias e mais disposto 
a trabalhar pelas melhorias propostas pela reitoria/administração, sem contar o maior engajamento para ensinar. O próprio engajamento pode, talvez, protegê-lo do Burnout, fato que ainda necessita de pesquisas longitudinais para se saber (TIJDINK JK, et al., 2014).

Sobre as limitações do estudo, cabe salientar que foi um estudo transversal, com um único centro médico acadêmico, onde não se obteve amostra total dos indivíduos. Não se dividiu em grupos os docentes médicos que somente ficam apenas em sala ou laboratório, daqueles que também prestam assistência hospitalar, e não foi realizado uma avaliação psicométrica da personalidade dos indivíduos, que poderia influenciar no entendimento e nos níveis de esgotamento. Existe um viés de resposta potencial, pois os participantes estavam no período da pandemia da Covid-19, em um contexto de valorização do profissional da saúde.

\section{CONSIDERAÇÕES FINAIS}

O Burnout entre os docentes representa um importante tema pois não somente impacta sua própria vida, mas também de seus alunos, residentes e o atendimento ao paciente. $O$ tema pode ser utilizado como instrumento de gestão da instituição direcionada ao docente médico, ao ensino e ao cuidado do paciente, ainda mais onde a atual pandemia trouxe mudanças na forma de trabalho às quais os docentes médicos precisaram se adaptar. Portanto, é de grande importância a identificação e programas de manejo de Burnout, havendo benefício em avaliar e gerenciar os processos de esgotamento no corpo docente médico, e a liderança institucional tem um papel fundamento para o seu bem-estar e valor.

\section{REFERÊNCIAS}

1. AMANULLAHS, SHANKAR RR. The impact of covid-19 on physician burnout globally: A review. Healthcare (Basel), 2020;8(4): 1-12.

2. AYDIN SAYILAN A, etal. Burnout levels and sleep quality of COVID-19 heroes. Perspectives in Psychiatric Care, 2021; 57(3): 1231-6.

3. ARVANDI Z, et al. Linking medical faculty stress/burnout to willingness to im plement medical school curriculum change: A preliminary investigation. Journal of Evaluation in Clinical Practice, 2016;22(1):86-92.

4. CHICHRA A, et al. Job stress and satisfaction in faculty of a teaching hospital in south India: A cross-sectional survey. Journal of Postgraduate Medicine, 2019;65(4): 201-6.

5. DANDAR VM, et al. Burnout among faculty in academic medicine. Analysis in Brief , 2019; (1): 1-2.

6. DIMITRIU MCT, et al. Burnout syndrome in Romanian medical residents in time of the COVID-19 pandemic. Medical Hypotheses, 2020;144.

7. DUKE NN, et al. Institutional Factors Associated With Burnout Among Assistant Professors. Te aching and Learning in Medicine, 2020;32(1):61-70.

8. GANESHAN D, et al. Burnout in Chairs of Academic Radiology Departments in the Un ited States. Academic Radiology, 2019;26(10): 1378-84.

9. GIUSTI EM, et al. The Psychological Impact of the COVID-19 Outbreak on Health Professionals: A Cross-Sectional Study. Frontiers in Psychology, 2020;1: 1-9.

10. KANNAMPALLIL TG, et al. Exposure to COVID-19 patients increases physician trainee stress and burnout. PLoS One, 2020;15(8): 1-12.

11. KNOX M, et al. Maslach Burnout Inventory and a Self-Defined, Single-ltem Burnout Measure Produce Different Clinician and Staff Burnout Estimates. Journal of General Internal Medicine, 2018;33(8): 1344-51.

12. LEITE TIA, et al. Prevalence and factors associated with burnout among university professors. Revista Bra sileira de Medicina do Trabalho, 2019; 17(2): 170-9.

13. LEITER MP, MASLACH C. Areas of Worklife: a Structured Approach To Organizational Predictors of Job Burnout. Research in Occupational Stress and Well Being, 2004; 3: 91-134.

14. LEITER MP, MASLACH C. Areas of Worklife Survey Manual and Sampler Set 5th Edition, 2011;1-32.

15. MASLACH C, et al. Maslach Burnout Inventory Manual, 2018; 81.

16. NASSAR AK, et al. Burnout among academic clinicians as it correlates with workload and demographic variables. Behavioral Sciences (Basel), 2020;10(6): 1-16.

17. PEREIRA SS, et al. Confirmatory factorial analysis of the Maslach Burnout Inventory - Human Services Survey in health professionals in emergency services. Revista Latino-Americana de Enfermagem, 2021;29: e3386. 
18. PORTO A. Áreas da Vida no Trabalho como preditoras da Síndrome de Burnout: tradução, adaptação transcultural e validação do modelo AWS-MBIGS [dissertação doutorado]. Santa Maria:Universidade Federal de Santa Maria, 2019.

19. RAO SK, et al. The impact of administrative burden on academic physicians: Results of a hospital-wide physician survey. Academic Medicine, 2017;92(2):237-43.

20. RAUDENSKÁ J, et al. Occupational burnout syndrome and post-traumatic stress among healthcare professionals during the novel coronavirus disease 2019 (COVID-19) pandemic. Best Practice \& Research Clinical Anaesthesiology, 2020;34(3): 553-60.

21. REED DA, et al. Effect of residency duty-hour limits: views of key clinical faculty. Archives of Internal Medicine,2007; (167): 1487-92.

22. RESTAURI N, SHERIDAN AD. Burnout and Posttraumatic Stress Disorder in the Coronavirus Disease 2019 (COVID 19) Pandemic: Intersection, Impact, and Interventions. Journal of the American College of Radiology , 2020;17(7):9216.

23. ROTENSTEIN LS, et al. Prevalence of burnout among physicians a systematic review. JAMA: The Journal of the American Medical Association, 2018;320(11):1131-50.

24. SCHINDLER BA, et al. The impact of the changing health care environment on the health and well -being of faculty at four medical schools. Academic Medicine, 2006;81(1):27-34.

25. TIJDINK JK, et al. Emotional exhaustion and burnout among medical professors; A nationwide survey. BMC Medical Education, 2014;14(1): 1-7.

26. VUKMIROVIC M, et al. The burnout syndrome in medical academia: Psychometric properties of the Serbian version of the maslach burnout inventory-educators survey. International Journal of Environmental Research and Public Health, 2020;17(16): 1-12.

27. WINDOVER AK, et al. Correlates and outcomes of physician burnout within a large academic medical center. JAMA: The Journal of the American Medical Association, 2018;178(6):856-8.

28. WU Y, et al. A Comparison of Burnout Frequency Among Oncology Physicians and Nurses Working on the Frontline and Usual Wards During the COVID-19 Epidemic in Wuhan, China. Journal of Pain and Symptom Management, 2020; 60(1): e60-5.

29. YU J, et al. Academic versus non-academic neurosurgeons in China: A national cross-sectional study on workload, burnout and engagement. BMJ Open, 2019; 9(10):1-9.

30. YU J, et al. Professional self-concept and burnout among medical school faculty in South Korea: A cross-sectional study. BMC Medical Education, 2019; 19(1): 1-6. 